\title{
LES RELATIONS ENTRE L'ACIDITÉ ACTUELLE ET L'ACIDITÉ POTENTIELLE DU LAIT
}

\author{
par M. A. TAPERNOUX
}

Chei des travaux de Chimie à l'Ecole Vétérinaire de Lyon

\section{L'acidité du lait}

Le seul lait pratiquement intéressant étant le lait de vache, c'est lui que nous prendrons comme type dans notre étude.

Nous envisagerons successivement:

10 La réaction du lait frais,

$2^{\circ}$ L'action des agents extérieurs sur la réaction du lait,

$3^{\circ}$ Les relations entre l'acidité potentielle et l'acidité actuelle du lait.

10 Réaction du lait frais. - Il nous semble utile avant d'exposer les résultats obtenus par les divers auteurs d'examiner avec un peu de détails la composition chimique du lait de vache frais.

$\mathrm{Si}$ nous nous reportons au travail du Professeur $\mathrm{Ch}$. Porcher et de A. Chevallier sur la répartition des matières salines dans le lait $(\mathbf{1})$ nous verrons que la composition moyenne du lait de vache peut s'exprimer par le tableau suivant (pour 1 litre de lait):

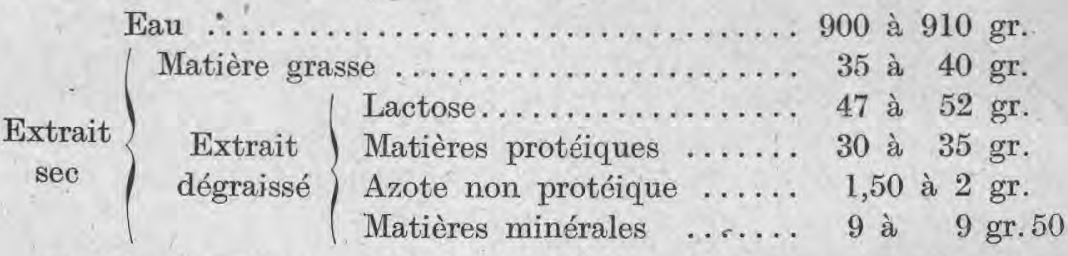

Si nous nous posons la question de savoir lesquelles de ces substances peuvent donner au lait une réaction déterminée, nous pouvons immédiatement éliminer l'eau, la matière grasse et le lactose.

La matière, grasse est, en effet, en suspension sous forme de globules plus ou moins volumineux qui ne peuvent avoir aucune influence sur la réaction du lait.

De son côté, le lactose en solution n'est susceptible d'amener aucune réaction particulière permettant la mise en liberté d'ions $\mathrm{H}^{+}$ou $\mathrm{OH}^{-}$, tout au moins pour le lait frais. Ce n'est qu'z̀ la suite de son dédoublement sous l'influence des ferments lactiques que le lactose intervient activement sur la réaction du lait.

Restent les autres composants du lait. Si nous les examinons tour à tour, nous voyons que chacun d'eux peut influencer la réaction du milieu : les matières protéiques (caséine, albumine, globuline) mettent

(1) Ch. Porcher \& A. Chevallier. Le Lait, 1923, no 2. 
en liberté des ions $\mathrm{H}^{+}$. ou $\mathrm{OH}^{-}$, les constituants de l'azote non pro téique parmi les acides aminés mettent également en liberté des ions $\mathrm{H}+$ et $\mathrm{OH}-$, et enfin les matières minérales peuvent aussi intervenir : de deux façons soit par inégalité entre les ions électropositifs et électronégatifs, soit par hydrolyse.

La part qui revient à l'azote non protéique dans la réaction du lait est faible, en raison de la concentration peu élevée des constituants réunis sous cette appellation; nous pouvons donc la négliger.l

Restent les matières protéiques et les matières minérales.

La plus importante des matières protéiques est la caséine dont le point isoélectrique est de $\mathrm{pH}=4,7$ et qui, par conséquent, amène dans le milieu plus d'ions $\mathrm{H}^{+}$que d'ions $\mathrm{OH}-$.

Les matières minérales que l'on pourrait plus exactement appeler matières salines sont constituées par les ions suivants:

Electronégatifs
Chlorhydrique
Phosphorique
Citrique
Carbonique
Sulfuriqúe

La plupart de ces ions ont été dosés directement. Cependant, jusqu'à ees temps derniers, le sodium était déterminé par différence; mais Barthe et Dufilho (1) ont pu doser directement cet ion en employant la méthode de Streng-Blanchetière (2).

Le dosage de l'ion carbonique est assez approximatif en raison de sa volatilité.

Il s'agirait de savoir le mode d'équilibre de tous ces ions en présence, compte tenu de leur action sur les matières protéiques du milieu. Il est bien évident que seule l'application de la loi d'action de masse, si elle était possible, nous déterminerait scientifiquement l'équilibre et nous donnerait la réaction du lait frais. La complexité des.ions en présence ne nous permet pas pour l'instant de résoudre le problème de cette façon. Des essais très intéressants ont été faits par Ch. Porcher et A. Chevallier(3) pour résoudre le problème par voie synthétique. Nous retiendrons seulement de leur travail les points suivants qui doivent fixer notre attention :

Une partie des ions calcium (environ $66 \%$ ) et une partie des ions phosphoriques sont unis à la caséine pour former un complexe appelé par Ch. Porcher : complexe caséina te dechaux + phosphate de chaux

(1) Bafthe \& DufilHo. Le Lait, nov. 1927.

(2) A. Blanchetrère. Bull. Soc. Chim. 9-4, 1923, pp. 807 et suiv.

(3) Porcher et Chevalifer. Loc. citato. 
d nt la consepsion nous est utile pour montrer les liens qui rattachent la caséine aux ions calcium et phosphorique, et nous serions étonné si ces rapports entre la caséine et les ions minéraux n'étaient pas étendus à d'autres ions.

Envisageons maintenant comment la question de l'acidité a été pratiquement étudiée.

Les auteurs s'accordent à reconnaître que suivant l'indicateur employé et la matière d'opérer, là réaction du lait frais est alcaline, amphotère ou acide. Ceci ne doit pas nous étonner, puisque nous avons vu que le propre des indicateurs colorés est de virer à des points différents de l'écholle des $\mathrm{pH}$. Ces considérations n'ont done qu'un intérêt historique. La premier, C. Foa(1) a recherchéle $\mathrm{pH}$ du tait de vache frais, c'est-à-dire mesuré son acidité réelle, et il démontra que la valeur du $\mathrm{pH}$ pouvait varier de 6,69 à 7,02 . Ceci nous montre que le lait a une réaction très voisine de la neutralité.

Ces ehiffres ont été confirmés par Van Slyke et Baker (2) qui adoptent les limites suivantes: 6,5 à 6,8 . Lours expériences faites sur plus de 300 échantillons provenant de vaches de rases différentes (Jersey H Jlstein-Friesan) ont montré que dans $83 \%$ des cas, le $\mathrm{pH}$ était compris entre 6,5 et 6,76 . Une petite proportion de laits individuels ont été trouvés moins acide, le $\mathrm{pH}$ pouvant atteindre 7,2. Leur expérimentation a porté sur des laits provenant de quartiers de mamelle et non sur le lait de la mamslle totale : il s'agit là de laits vraiment individuels.

Il est donc facile de concevoir qu'en se servant d'un indicateur dont le $\mathrm{pH}$ de virage est inférieur à 6,5 , le lait apparaîtra comme alcalin. C'est ce qui se produit lorsqu'on se sert d'hélianthine qui vire définitivement au jaune pour $\mathrm{pH}=4,4$.

Au eontraire, si le $\mathrm{pH}$ de virage de l'indicateur est supérieur à 7 , le lait apparaîtra comme acide. C'est ainsi qu'il se comporte vis-à-vis de la phtaléine du phénol qui, nous le savons, commence à virer pour un $\mathrm{pH}=8,2$.

Las méthodes titrimétriques de détermination de la réaction du lait utilisent pratiquement la phtaléine du phénol comme indicateur. Il va donc de soi, dans ce cas, de parler de l'acidité du lait.

a) Méthodes de titration de l'acidité du lait. - Leur prineipe se résume pourtoutes dans l'addition au lait préalablement mélangé avec l'indicateur, de quantités progressives de soude titrée jusqu'à apparition d'une teinte rose nettement perceptible.

Les modalités de l'opération et l'expression des résultats changent suivant les pays. Nous reproduisons une rapide description des différents procédés employés :

10 Procéd' français. Cuurammənt désigué du |nom de proeédé Dor-

(1) C. Foa. C. R. Soc. de Biol. $1^{\text {er }}$ juillet 1905.

(2) VAN SLYKE et BAKER. Technical Bull, n० 70. New-York Agricultural Experiment Station. Tuin 1919, pp. 2 et 3. 
nic, est, à notre avis, le plus intéressant en même temps que le plus clair dans l'expression des résultats.

On opère sur $10 \mathrm{ec}$. de lait additionnés de deux gouttes d'une solution de phénolphtaléine à $2 \%$ dans l'alcool à $75^{\circ}$. La soude titrée est exactement $\mathrm{N} / 9$.

L'expression des résultats s'effectue en degrés Dornic (D) qui représentent le nombre de $1 / 10$ de centimètres cubes nécessaires à faire apparaître la teinte rose de la phtaléine. Un appareil spécial imaginé par Dornic permet d'effectuer rapidement un grand nombre de déterminations.

L'auteur du procédé a choisi le titre N/9 de la soude pour qu'une simple transposition de virgule permette d'exprimer l'acidité du lait en acide lactique. Le degré Dornic, en effet, représente le nombre de décigrammes d'acide lactique que conventionnellement ont dit équivaloir l'acidité d'un litre de lait.

L'acidité du lait frais et normal est de 15 à $16^{\circ} \mathrm{D}$. qui correspondent, à 1 gr., 5 - 1 gr., 6 par litre. Cette évaluation répétons-le n'est qu'une fiction: l'acidité originelle du lait n'est pas due à l'acide lactique.

$2^{\circ}$ Procédé Soxhlet-Henckel. - C'est le premier en date. On opère sur une quantité de lait variable $(25$ cc., 50 cc., 100 cc. mais en rapportant les résultats à $100 \mathrm{ce}$. On emploie de la soude titrée $\mathrm{N} / 4$. On se sert de la même solution d'indicateur à la dose de 4 ce. pour $100 \mathrm{ce}$. de lait.

Les résultats sont exprimés en Degrés Soxhlet-Henckel (S-H) qui représentent le nombre de centimètres cubes de soude nécessaires pour neutraliser $100 \mathrm{cc}$. de lait.

On peut vérifier que

$$
\text { Degré Dornic }=\frac{\text { Degré Soxhlet-Henckel. }}{2.25}
$$

Le procédé de Peter est une variante du précédent; il opére sur $10 \mathrm{cc}$. de lait. Le nombre de $1 / 10 \mathrm{c} / \mathrm{de} \mathrm{cm}^{3}$ de soude $\mathrm{N} / 4$ employé indique le degré Peter. lequel est identique au degré Soxhlet-Henckel.

Les autres procédés (Pfeiffer, Thörner) utilisent $10 \mathrm{cc}$. de lait addiltionnés d'eau distillée et de soude décinormale. Le nombre de 1/10 de $\mathrm{cm}^{3}$ employés expriment le degré Pfeiffer ou Thôrner. Ces dernières méthodes devraient être abandonnées. On a, en effet, remarqué, que lorsqu'on ajouté à $10 \mathrm{cc}$. de lait, de l'eau en plus ou moins grande quantité, la quantité de soude titrée nécessaire pour provoquer le virage de la phtaléine diminue. Il y a un déplacement de l'acidité potentielle avec virage vers l'alcalinité. Il est facile d'expliquer ce phénomène par le jeu de la loi d'action de masse : la réaction du lait n'est pas déter- 
minée par la présence d'un acide, mais par l'équilibre d'un certain nombre d'ions à une concentration déterminée. Que cette concentration vienne à varier et l'équilibre primitif sera remplacé par un autre qui dépendra des nouvelles concentrations des ions en présence. Il est donc indiqué de ne jamais additionner d'eau la quantité primitive de lait nécessaire à la titration acidimétrique.

Il est également à noter qu'on détermine l'acidité du lait à froid et dans ces conditions, l'acide carbonique libre du lait intervient puisque la phénolphtaléine est sensible à cet acide.

Enfin, on peut constater que lorsque la teinte rose a été obtenue, elle disparaît au bout de quelques minutes, le lait reprenant sa couleur blanche primitive. Quelle est l'explication de ce phénomène? D'aucuns ont prétendu qu'il avait pour cause la fixation de l'acide carbonique de l'air. Nous ne le pensons pas, car si l'on fait bouillir le lait ainsi traité et redevenu blanc, la teinte rose ne réapparaît pas. Bien mieux, si on porte à l'ébullition du lait préalablement neutralisé, et présentant encore la teinte rose, celle-ci disparaît.

On pourrait peut-être penser que la saponification de la matière grasse du laitintervient dans la production de ce phénomène, maisil n'en est rien, car la même réaction s'observe avec les laits complètement écrémés.

Il y a lieu de faire intervenir ici la caséine. En effet, cette matière protéique s'unit lentement aux bases pour donner des caséinates, et, lorsqu'on titre l'acidité d'un lait, l'opération est assez rapide, ce qui ne permet pas à la réaction caséine + base de s'effectuer d'une façon définitive. En un mot, l'équilibre n'est pas atteint lorsque la phtaléine du phénol a subi un premier virage. Nous trouverons des éléments de justification de cette opinion lorsque nous étudierons l'acidité de là caséine.

b) Méthodes de mesure de I'acidité actuelle ou du pH du lail.

10 Méthode électrométrique. - Cette méthode est applicable à la détermination $\mathrm{du} \mathrm{pH}$ du lait. Elle est exacte lorsque les opérations sont convenablement conduites, mais elle nécessite la possession de nombreux appareils dont la liste suit :

$1^{\circ}$ Un accumulateur,

$2^{\circ}$ Un potentiomètre,

$3^{\circ}$ Des électrodes,

$4^{0}$ Une source d'hydrogène lorsqu'on utilise des électrodes d'hydrogène.

L'accumulateur $\mathbf{P}$ est destiné à équilibrer la différence de potentiel aux deux électrodes, sa force électromotrice doit être voisine de 2 volts.

Le principe du potentiomètre repose sur la méthode de compensation de Pogendorff. Les deux électrodes $\mathrm{E}$ et $\mathrm{K}$ sont en contact avec les deux bornes A et B sur lesquelles sont également branchées les 
deux bornes de l'accumulateur munies d'un rhéostat $\mathrm{R}$ et.d'un millivoltmètre $M V$. Les deux connexions sont faites de telle sorte que l'électrode positive et le pole positif de l'accumulateur soient en relation avec le même borne A. Les deux bornes A et $\mathrm{B}$ sont également reliées entre elles par un conducteur A B muni d'un galvanomètre $G$ permettant d'apprécier le passage du courant entre A et B, ou l'absence de courant au moment où la force électromotrice développée par les électrodes est équilibrée par celle $q u_{i}$ provient de l'accumulateur. Pour effectuer une détermination, on fait varier la résistance

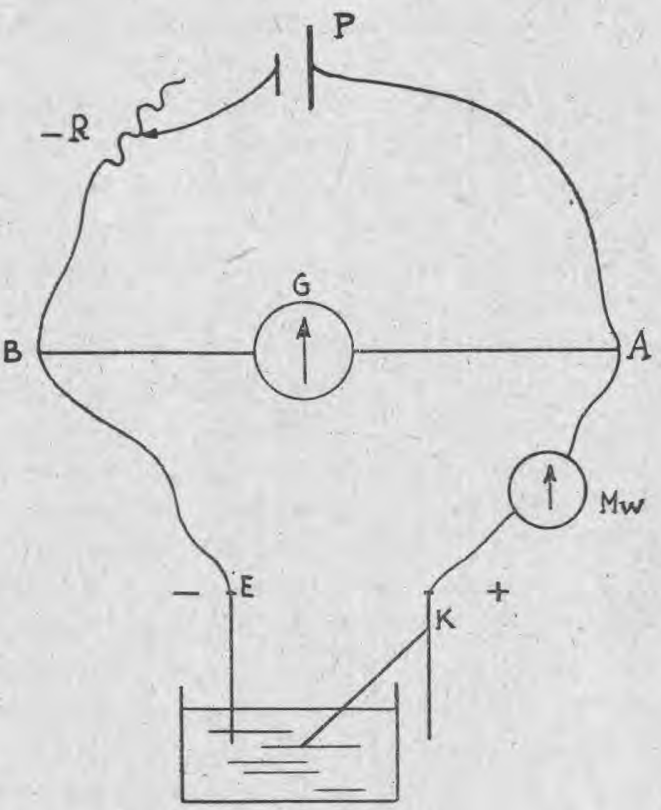
$R$ jusqu'au moment où aucun courant ne passe entre A et B, ce qu'il est facile de constater à l'aide du galvanomètre G. On mesure alors la force électromotrice à l'aiguille du millivoltmètre. On peut utiliser à la place du galvanomètre G un instrument de plus grande précision, l'électromètre capillaire.

Les électrodes employées appartiennent à trois catégories :

10 Les électrodes d'hydrogène,

$2^{\circ}$ L'électrode de quinhydrone,

$3^{\circ}$ L'électrode au calomel.

Les électrodes d'hydrogène, constituées par une masse de platine recouverte électrolytiquement de noir de platine, sont de différents modèles, suivant les auteurs qui les ont conçueset l'usage auquel elles sont destinées.

Nous sommes particulièrement satisfait pour le lait de l'électrode de Hildebrandt, qui plonge directement dans le liquide à étudier, et dans laquelle le courant d'hydrogène arrive par un tubulure latérale (figure 1 ).

L'électrode d'hydrogène a parfois des inconvénients lorsque le liquide étudié peut être modifié dans sa composition par le cburant d'hydrogène nécessaire. Pour le lait, il n'est pas à craindre un effet de 
réduction, mais le courant d'hydrogène peut déplacer et entraîner une partie de l'acide carbonique dissous.

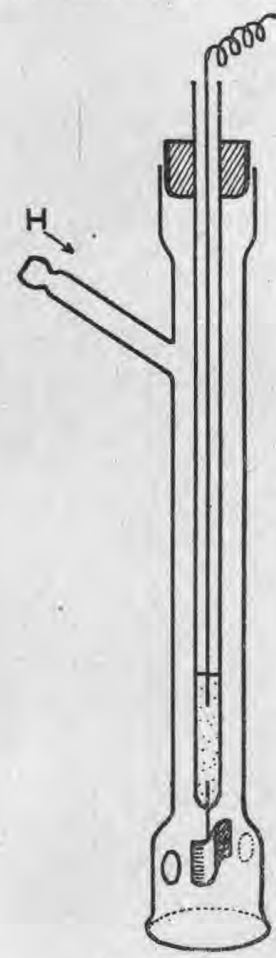

Figure 1

Il est alors indiqué de se servir de l'électrode de quinhydrone qui remplace l'électrode d'hydrcgène avec une légère modification de montage. Cette électrode est essentiellement formée par une palette de platine non platiné qui plonge dans le liquide à étudier. On ajoute à ce liquide gros comme un pois de quinhydrone, et on agite avec la palette de platine préalablement nettoyée à l'acide sulfurique et à l'eau distillée, puis séchée à la flamme d'une lampe à alcool. Le liquide à étudier est relié par un pont de chlorure de potassium à l'électrode au calomel. En quelques minutes la lecture est possible.

L'électrode de quinhydrone a sur l'électrode d'hydrogène l'avantage de la rapidité. De plus, elle ne nécessite pas de source d'hydrogène. Nous verrons qu'elle n'est malheureusement applicable que pour des solutions dont le $\mathrm{pH}$ ne dépasse pas 7, c'est-à-dire pour les solutions acides. Pour l'étude du lait de vache, cette électrode est l'électrode de choix, et peut facilement être utilisée. Nous n'avons noté aucun inconvénient résultant de son emploi (figure 2).

L'électrode de calomel, due à Michaelis et Davidoff (1912), faitpartie de toute installation électrométrique, et s'emploie toujours soit contre l'électrode d'hydrogène, soit contre l'électrode de quinhydrone. Elle est essentiellement constituée par une bouillie de mercure mélangé à du calomel, au-dessus de laquelle se trouve une solution de chlorure de potassium de titre connu. Pour le lait, nous avons toujours employé l'électrode de calomel saturée, c'est à-dire l'électrode formée avec une solution saturée de chlorure de potassium Le contact avec le circuit a lieu par le mercure, comme l'indique

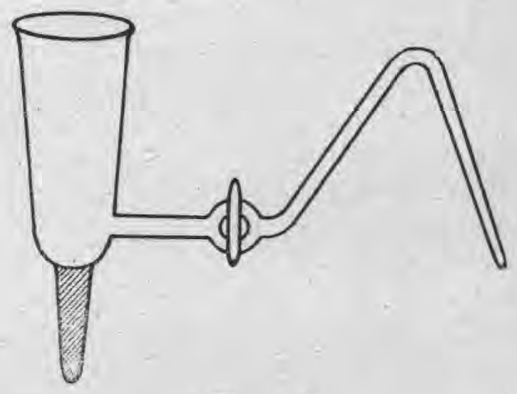

Figure 2 la figure, pendant qu'une tubulure latérale remplie par la solution de chlorure de potassium plonge dans le liquide à étudier. On peut également employer des électrodes au calomel préparées 
avec des solutions $\mathrm{N}$ ou $\mathrm{N} / 10$ de chlorure de potassium figure 3 ).

Lorsqu'on emploie l'électrode au calomel contre l'électrode d'hydrogène, la première doit être reliée au pole positif du potentiomètre, et la seconde au pôle négatif. Dans ces conditions si E représente en millivolts la force électromotrice lue au millivoltmètre $a$ et $b$, deux constantes dont les valeurs sont données par le tableau, nous avons

$$
\mathrm{pH}=\frac{\mathrm{E}-\mathrm{a}}{\mathrm{b}} .
$$

La valeur de E. se détermine aisément, mais l'équilibre est long à obtenir. Il faut veiller à ce que la pression du courant $d$ 'hydrogène soit bien constante, ce que l'on voit facilement en

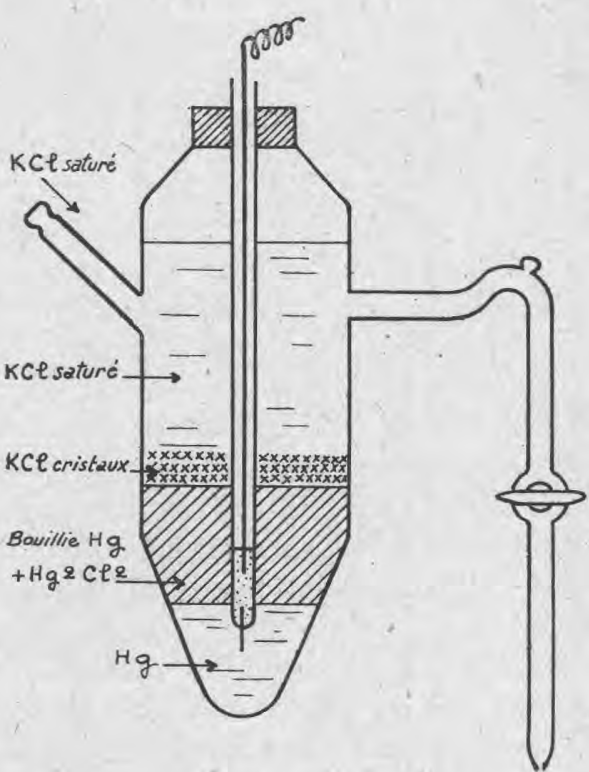

Figure 3 notant la régularité de la formation des bulles dans le liquide. De plus, on ne fera la lecture que lorsque trois déterminations successives, à cinq minutes d'intervalle auront donné la même valeur pour $\mathrm{E}$. A /ce moment l'équilibre est atteint :

$\begin{array}{ccc}\text { Température } & \mathrm{a} & \mathrm{b} \\ 15^{\mathrm{o}} & 252,5 & 57,1 \\ 16 & 251,7 & 57,3 \\ 17 & 250,9 & 57,5 \\ 18 & 250,3 & 57,7 \\ 19 & 249,5 & 57,9 \\ 20 & 248,8 & 58,1 \\ 25 & 245,5 & 58,7\end{array}$

Enfin,il faut noter que le maniement de l'électrode d'hydrogène est extrêmement délicat. Celle-ci doit être souvent replatinée; elle peut souvent mal fonctionner pour de multiples raisons dont l'intervention échappe à l'opérateur. Dans le cours de mes expériences personnelles, j'ai constaté pendant deux jours des résultats anormaux dont la cause était que, pendant le platinage de mon électrode, le fil de cuivre reliant l'autre électrode à la pile avait légèrement trempé dans une solution 
de chlorure de platine : un peu de cuivre s'était déposé sur mon électrode d'hydrogène.

Lorsqu'on emploie l'électrode de calomel contre l'électrode de quinhydrone, il faut relier la première au pôle négatif du potentiomètre, et la seconde au pôle positif. Dans ces conditions la formule donnant le $\mathrm{pH}$ est la suivante :

$$
\mathrm{pH}=\frac{451-\mathrm{E}}{57,7}
$$

On voit immédiatement que lorsque $\mathrm{E}=451$ millivolts, $\mathrm{pH}=\mathrm{O}$, e'est le cas de la solution normale acide.

$$
\text { Lorsque } \mathrm{E}=0 \text { millivolt } \quad \mathrm{pH}=7,81
$$

L'électrode de quinhydrone permet donc la détermination du $\mathrm{pH}$ entre les limites 0 et $7 ; 81$.

J'ai essayé d'utiliser l'électrode de quinhydrone pour mesurer le $\mathrm{pH}$ des solutions alcalines en effectuant la transformation suivante:

$1^{0}$ Pour les solutions de $\mathrm{pH}$ supérieur à 7,81 , je change l'électrode de quinhydrone de pôle en la permutant avec l'électrode de calomel. L'électrode de quinhydrone est alors reliée au pôle négatif et l'électrode au calomel au pôle positif du potentiomètre.

$2^{\circ} \mathrm{Je}$ modifie la formule qui devient:

$$
\mathrm{pH}=\frac{451+\mathrm{E}}{57,7}
$$

J'ai vérifié sur deux caséinates de chaux que ladite mesure était possible comme en témoignent les résultats suivants :

$$
1^{\text {re Expérience. }}
$$

$\mathrm{pH}$ du Ier caséinate de chaux mesuré à l'électrode d'hydrogène

$\mathrm{pH}$ du même caséinate mesuré à l'électrode de quinhydrone

$$
2^{\text {me }} \text { expérience. }
$$

$\mathrm{pH}$ du $2^{\mathrm{e}}$ caséinate mesuré à l'électrode d'hydrogène

$\mathrm{pH}$ du même caséinate mesuré à l'électrode de quinhydrone $\quad 8,5$

En ce qui concerne la source d'hydrogène, il est nécessaire que l'hydrogène utilisé soit pur et, d'autre part, que sa pression soit constante. On peut très bien se servir d'un appareil continu (modèle Kipp) et produire l'hydrogène par l'action de l'acide sulfurique pur et dilué sur le zine pur. On obtient une meilleure constance dans la pression en utilisant un obus à hydrogène muni d'un appareil de détente.

$2^{\circ}$ Méthode colorimétrique. - Cette méthode, nous le savons, réside 
dans l'appréciation comparée de la teinte d'un indicateur mis au contact de la solution dont on veut connaître le $\mathrm{pH}$ avec la teinte obtenue dans les mêmes conditions au moyen de solutions étalons d'un $\mathrm{pH}$ déterminé. Cette méthode donne des résultats pratiquement suffisants lorsque les solutions étudiées sont limpides. Malheureusement il n'en est pas de même pour le lait milieu opaque, en raison de sa composition.

Dans le cas du lait, les indicateurs colorés donnent des résultats très irréguliers.

De nombreux auteurs ont essayé d'obtenir à partir du lait des solutions limpides permettant l'utilisation des indicateurs :

Schultz et Chaudler (1) proposent la dialyse du lait au travers d'un sac de collodion dans des conditions déterminées.

Hirsch (2) recommande la précipitation du lait par l'oxyde ferrique colloïdal.

Vandervelde(3) obtient un sérum limpide en traitant $100 \mathrm{cc}$, de lait par 1 cc. d'une solution N/2 de chlorure de baryum et $99 \mathrm{cc}$. d'alcool neutre à $94^{\circ}$.

On doit s'élever contre de pareilles méthodes L'acidité du lait,est le résultat d'un équilibre, et il nous semble illogique lorsqu'on veut avoir la physionomie de cet équilibre, de commencer par le modifier, sans fixer au préalable d'une façon absolue les conditions, et surtout les résultats de cette modification.

Nous avons montré qu'il existe une méthode relativement simple de détermination précise $\mathrm{du} \mathrm{pH}$ du lait: la méthode électrométrique avec emploi de l'électrode de quinhydrone. Devons-nous recourir à une méthode moins précise en nous servant de procédés qui dénaturent le liquide que nous voulons étudier ? Nous ne le pensons pas et nous serions fort étonné si le problème envisagé sous cet angle se trouvait un jour résolu.

Est-ce à dire que la méthode colorimétrique soit complètement à rejeter pour le lait ? Telle n'est pas ma pensée, et des résultats encourageants ont été obtenus par les auteurs. qui ont abordé le problème franchement.

Morres, en 1911(4) a employé la solution alcoolique d'alizarine pour évaluer la fraîcheur d'un lait. Dans une étude extrêmement intéressante, Valencien et Panchaud(5) ont fait connaître les applications de ee procédé et les différentes teintes exposées, suivant la qualité du lait. La solution employée est une solution alcoolique dans l'alcool neutre à $68^{\circ}$, au titre de 1 gr., 5 à 2 gr. par litre. La teinte normale de

(1) Sohultz et Chaudler. Journ. Biol. Chem. T. 46, pp. 129-131.

(2) Hrrsch. Biochem. Zeitschr. 1924, 147, p. 423.

(3) VANDEVEnde. Le Lait, 1927, no 62, p. 143.

(4) Zeitsch. Unters. Nahrung. 1911, 22, p. 459.

(5) Valencien et Panohaud. Le Lait. 1923, no 7-8, p. 523. 
cette solution est vieux bordeaux. Pour pratiquer un essai, on mélange dans un tube à essai $3 \mathrm{cc}$. de lait et $3 \mathrm{cc}$. de solution précédente, et on compare la teinte obtenue à celles fixées sur un tableau. Je laisse völontairement de côté les renseignements fournis par la coagulation du liquide étudié sous l'influence de l'alcool. L'intervalle de virage de l'alizarine est compris entre $\mathrm{pH}=5,5$ et $\mathrm{pH}=6,8$ : la matière colorante passe du jaune au violet

On peut également se servir de l'alizarine sulfonate de sodium qui donne les mêmes indications.

Baker et Van Slyke (1) ont utilisé le pourpre de bromocrésol ou dibromocrésolsulfophtaléine, dont l'intervalle de virage est compris entre $\mathrm{pH}=5,2$ et $\mathrm{pH}=6,8$. La coloration passe du jaune au bleu-violet.

Kolthoff $(2)$ emploie le rouge de phénol ou phénolsulfophtaléine dont l'intervalle de virage est compris entre $\mathrm{pH}=6,8$ et $\mathrm{pH}=8,4$. La coloration passe du jaune au rouge.

Cooledge(3) a préconisé pour étudier le développement de l'acidité dans les cultures, le bleu de bromothymol, ou dibromothymolsulfophtaléine, dont l'intervalle de virage est compris entre $\mathrm{pH}=6$ et $\mathrm{pH}=7,6$. La coloration varie du jaune au bleu. J'ai moi-même étudié cet indicateur en préparant la solution suivante : on broie au mortier 0 gr., I de bleu de bromothymol et on ajoute goutte à goutte $3 \mathrm{ce}$. de soude $\mathrm{N} / 20$, puis on complète avee de l'eau à 250 cc.

Dans ces conditions, un lait frais normal donne une coloration réséda lorsqu'on l'additionne de son volume de la solution précédente.

Enfin, signalons que l'aeide rosolique a parfois été utilisé. Cet acide qui constitue un mélange assez complexe a un intervalle de virage compris entre $\mathrm{pH}=6,9$ et $\mathrm{pH}=8$. Il vire du jaune au rouge. Schmidt(4) conseille d'ajouter à $10 \mathrm{ce}$. de lait, $10 \mathrm{cc}$. d'alcool à $96^{\circ}$, et quelques gouttes d'une solution d'acide rosolique dans l'eau distillée à $1 \%$. Le lait normal et frais prend une teinte jaune-brun.

Dans une communication antérieure (5)j'ai fait connaître l'utilisation possible du rouge de méthyle, ou acide diméthylaminoazo-benzène -ocarbonique dont l'intervalle de virage est compris entre $\mathrm{pH}=4,4$ et $\mathrm{pH}=6,2$. La coloration varie du rouge au jaune. J'a préparé avec cet indicateur un papier qu'il suffit de tremper dans le lait pour avoir une idée de sa qualité. Avec le lait normal et frais, le papier reste jaune.

De toutes ces méthodes, il faut retenir qu'elles ne donnent et ne peuvent donner que des résultats approximatifs concernant la valeur

(1) Journal of Biol. Chem: 1919, 40, p. 335 .

(2) Handelingen Genootschap Melkkunde, 1920.

(3) Cooledge. Agr. Experiment Station, Michigan. Bull. 52, nov. 1922.

(4) Gerber et Otmiker. Traité pratique des essais du lait, p. 88.

(5) A. Tapernoux. C. P. Soc. Biologie. 21 février 1928. 
du $\mathrm{pH}$. Il faut également retenir que les renseignements qu'elles fournissent sont amplement suffisants en pratique, et, par conséquentextrêmement intéressants. En particulier, l'utilisation du papier indieateur permet une évaluation rapide de l'acidité du lait en dehors de tout laboratoire et de toute instrumentation.

\section{Action des agents extérieurs sur la réaction du lait.}

Nous venons d'envisager la réaction du lait qu'on peut dire normal, et immédiatement après la traite. Cette réaction peut varier sous l'influence d'un certain nombre de causes que nous allons étudier une à une, et cette étude nous permettra de suivre l'évolution de la réaction du lait pendant la période où agissent les facteurs susceptibles de la modifier. Nous noterons, au préalable, que chacune de ces causes peut agir seule, ou en combinaison avec toutes les autres, amenant ainsi une nouvelle complexit dans l'étude de la question.

\section{a) Action microbiennes.}

Nous les étudierons en premier lieu parce qu'elles sont les plus importantes et qu'elles se produisent spontanément. Nous supposons que le lait soumis à notre étude est normal à la traite, au sens de la loi du 1 er août 1905 sur la répression des fraudes. Nous réservons pour un autre chapitre l'étude des laits malades.

Nous étudierons successivement:

$1^{\circ}$ La fermentation lactique,

$2^{\circ}$ La putréfaction.

$1^{\circ}$ Fermentation lactique. - Le lait, même au moment de la traite contient déjà des microbes; que ceux-ci proviennent du canal du trayon de l'animal, des mains du vacher, des ustensiles ou de manipulations plus ou moins aseptiques. Parmi eux, il en est qui sont très variables dans leur morphologie: $e^{\prime}$ est le groupe des ferments lactiques. Bornant notre étude seulement aux actions, et non aux formes microbiennes, nous pouvons dire que, sitôt après la traite, ces microbes se développent dans le lait en provoquant une altération du milieu et une rupture de son équilibre antérieur. Leur développement se produit, en effet, aux dépens du lactose, substance neutre à partir de laquelle, par des processus à la fois hydrolytiques et dédoublants, les microbes provoquent la formation d'acide lactique, modifiant ainsi la concentration en ions hydrogène du lait. Nous exprimerons cette transformation par l'équation:

$$
\mathrm{C}^{12} \mathrm{H}^{22} \mathrm{O}^{11}+\mathrm{H}^{2} \mathrm{O}=4 \mathrm{C}^{3} \mathrm{H}^{6} \mathrm{O}^{3}
$$

L'acidification du lait sous l'influence des ferments lactiques est un phénomène constant et naturel, de telle sorte que si, à la traite, le 
lait ne contient pas d'acide lactique, peu après il en renferme toujours, ce qui rend logique la détermination de l'acidité du lait en acide lactique.

Sous cette influence, l'acidité de titration du lait augmente, ainsi que son acidité réelle, avee le nombre des microbes qu'il contient.

Cette action est plus ou moins rapide suivant la température à laquelle est soumise le lait. Très lente au voisinage de $0^{\circ}$ centigrade, elle augmente d'intensité et de rapidité jusqu'à un optimum de $37^{\circ}$, température qui, nous le savons, est la plus favorable à la culture des ferments lactiques ordinaires.

(A suivre.)

\title{
L'INFLUENCE DE LA CASÊINE EN SUSPENSION, LORS DU BARATTAGE DE LA CRËME ACIDIFIEE
}

\author{
par W. van DAM et B. J, HOLWERDA
}

Les recherches dont les données sont communiquées dans ce travail, furent d'abord faites dans le but suivant. Selon la théorie de RAHN (1) sur le processus de barattage, la modification de la teneur en caséine, lors de barattage de crème acidifiée, n'a aucune influence, ou certainement bien moins d'influence que celle de la teneur en albuminoïdes en solution. Cette dernière modification a de l'influence, notamment sur la formation d'écume et sur la tension superficielle. En premier lieu fut examinée l'influence de la quantité de la easéine en suspension; mais les résultats obtenus étaient tellement inattendus, que nous avons décidé de continuer nos recherches sur ce point, bien que de cette façon, nous nous écartions, dans certaine mesure, du but primitif de nos recherches. Cette décision fut d'ailleurs encore influencée par d'autres circonstances imprévues.

Pour observer l'influence sur l'évolution du processus du barattage, de quantités diverses de caséine, il fallait des expériences parallèles dans lesquelles, seules, les quantités de caséine étaient différentes, tandis que tous les autres facteurs : teneur en matière grasse, degré d'acidité, conditions de la matière grasse, etc. étaient identiques. Pour réaliser ces conditions, il fut procédé de la façon suivante. Du lait fut centrifugé de façon à obtenir de la crème contenant plus de $40 \%$ de matière grasse et du lait écrémé à teneur en matière grasse aussi réduite que possible. Les deux produits ainsi obtenus furent pasteurisés, par le maintien pendant 25 minutes à la température de $65^{\circ} \mathrm{C}$. et après réfrigération, la crème fut placée dans de la glace pendant 22 heures au moins, tandis que le lait écrémé fut acidifié par l'addition d'une culture pure.

(1) Forschungen auf dem Gebiete der Milehwirtschaft und des Molkereiwesens. I, 309 (1921). 